\title{
Immune cell-derived small extracellular vesicles in cancer treatment
}

\author{
Sung-Jin Choi ${ }^{1, \#}$, Hanchae Cho ${ }^{2, \#}$, Kyungmoo Yea ${ }^{1, *} \mathcal{E}$ Moon-Chang Baek ${ }^{2, *}$ \\ ${ }^{1}$ Department of New Biology, DGIST, Daegu 42988, Republic of Korea, ${ }^{2}$ Department of Molecular Medicine, CMRI, Exosome Convergence \\ Research Center (ECRC), School of Medicine, Kyungpook National University, Daegu 41944, Republic of Korea
}

Small extracellular vesicles (sEVs) secreted by most cells carry bioactive macromolecules including proteins, lipids, and nucleic acids for intercellular communication. Given that some immune cell-derived sEVs exhibit anti-cancer properties, these sEVs have received scientific attention for the development of novel anticancer immunotherapeutic agents. In this paper, we reviewed the latest advances concerning the biological roles of immune cell-derived sEVs for cancer therapy. sEVs derived from immune cells including dendritic cells (DCs), T cells, natural-killer (NK) cells, and macrophages are good candidates for sEV-based cancer therapy. Besides their role of cancer vaccines, DC-shed sEVs activated cytotoxic lymphocytes and killed tumor cells. sEVs isolated from NK cells and chimeric antigen receptor (CAR) T cells exhibited cytotoxicity against cancer cells. SEVs derived from $\mathrm{CD8}^{+} \mathrm{T}$ and $\mathrm{CD4}^{+} \mathrm{T}$ cells inhibited cancer-associated cells in tumor microenvironment (TME) and activated $B$ cells, respectively. M1-macrophage-derived sEVs induced M2 to M1 repolarization and also created a pro-inflammatory environment. Hence, these $\mathrm{sEVs}$, via mono or combination therapy, could be considered in the treatment of cancer patients in the future. In addition, $\mathrm{sEV}$ s derived from cytokine-stimulated immune cells or $\mathrm{SEV}$ engineering could improve their anti-tumor potency. [BMB Reports 2022; 55(1): 48-56]

\section{INTRODUCTION}

Basics: biogenesis, secretion, and uptake of sEVs

Over the past decades, small extracellular vesicles (sEVs) have received considerable scientific attention due to their role in

*Corresponding authors. Moon-Chang Baek, Tel: +82-53-420-4948; Fax: +82-53-421-4970; E-mail: mcbaek@knu.ac.kr; Kyungmoo Yea, Tel: +82-53-785-1760; Fax: +82-53-785-1819; E-mail: ykm31@dgist.ac.kr ${ }^{\#}$ These authors contributed equally to this work.

https://doi.org/10.5483/BMBRep.2022.55.1.133

Received 26 August 2021, Revised 6 October 2021, Accepted 26 October 2021

Keywords: Cancer-immune cycle, Cancer immunotherapy, Exosomes, Immune cells, Small extracellular vesicles cell-cell communication and their potential in disease treatment. sEVs are in the range of $\sim 40-200 \mathrm{~nm}$ in diameter $(1,2)$ and are produced by almost all types of cells and exist in many biological fluids such as blood, urine, breast milk, and saliva $(3,4)$. Since $s E V s$ exert diverse functions on the basis of their cell origin, it is important to understand their basic mechanism in order to interpret their function and for future application.

$\mathrm{sEVs}$ are generated via a process involving the infolding of the plasma membrane, followed by the organization of multivesicular bodies (MVBs) which contain intraluminal vesicles (ILVs). Then, MVBs fuse with the plasma membrane and the ILVs are ultimately secreted into the extracellular space as sEVs (2). Several proteins, such as Ras-related protein (Rab) GTPase, Sytenin-1, tumor susceptibility gene 101 (TSG101), apoptosislinked gene 2-interacting protein X (ALIX), and syndecan-1, are involved in the biogenesis of sEVs (5-7). In the generation process, various cargos including proteins and nucleic acids are assembled into sEVs (8). Since the cargos and their membranes exhibit unique properties of $\mathrm{sEVs}$, the similarity in the components between parent cells and their sEVs is important in the investigation of $\mathrm{sEV}$ function.

The secretion of $\mathrm{sEV}$ s follows an ordered series of events involving intracellular transport and plasma membrane fusion with MVBs. These processes undergo a specific coordination of soluble N-ethylmaleimide-sensitive factor attachment protein receptors (SNAREs), Rab GTPase, integrins, tetraspanins, and cytoskeletons, which are localized on either MVBs or intracellular side of the plasma membrane. sEV release is governed by extracellular stimuli (9). For example, dendritic cells (DCs) and $B$ cells show reinforced $s E V$ secretion after interaction with $T$ cells or senescence induction $(10,11)$. Also, mature DCs secrete more strengthened $\mathrm{sEV} s$ as compared to immature DCs (11). Even if sEVs are secreted from the same type of cell, the quantity or components of $\mathrm{sEV}$ s differ depending on the cell status. Therefore, understanding the secretion procedure is important to characterize sEVs for biomarker and for harvest of $s E V s$ for therapeutic application.

sEV uptake is the last stage involving transportation of components to recipient cells (5). After secretion, circulating sEVs are recruited into nearby cells in various organs and tissues via 4 different pathways, namely, ligand-receptor interaction, cleavage of membrane proteins that bind to target receptors, mem- 
brane fusion, and endocytic mechanisms (9). Each route needs respective membrane proteins. For example, there are highly enriched integrins on $\mathrm{SEV}$ surface from B cells which enhance sEV fusion with antigens (12). Also, intercellular adhesion molecule 1 (ICAM1) located on mature DC-derived sEV surface specifically interacts with the lymphocyte function-associated antigen 1 (LFA-1) receptor and thus allow sEVs to enter the antigen-presenting cells (APCs) (13). Consequently, in the aspect of specific targeting, further studies about the uptake mechanism and related biomolecules would provide more insight in engineering of sEVs for use as drug vehicles in therapeutics.

\section{Promising roles of sEVs in immuno-oncology}

sEVs have extensive and unique advantages in diagnosis and application compared to synthetic carriers and cell-therapy. Due to the fact that $s E V s$ are small vesicles which originate from living cells, they possess low immunogenicity, high surface/ volume ratio, and unique delivery properties $(2,14,15)$. Although there are still some drawbacks such as low stability and yield, sEVs, with the help of engineering and isolation technique, are expected to be promising natural carries containing bioactive molecules for clinical applications (16).

In particular, recent reports focus on the use of sEVs for cancer immunotherapeutic applications (16-18), owing to the unique characteristics of tumor microenvironment (TME). The TME typically comprises of tumor cells, immune cells, and stromal cells, such as pericytes, mesenchymal stromal cells (MSCs), and cancer-associated fibroblasts (CAFs). In TME, im- mune cells detect and attack tumor cells through antigenspecific and nonspecific mechanisms and simultaneously activate other immune cells by transferring neoantigens (19). On the other hand, the stroma cells promote the TME remodeling, thereby suppressing the immune cell infiltration and enhancing immune surveillance and metastasis (20). Since these dynamic reciprocal communications are conducted via secreted molecules as well as cell-cell interaction, understanding the relation between distant cell in TME is crucial for cancer treatment. Given their ability to mimic the function of origin cells and easily penetrate the extracellular matrix (ECM), sEVs have received scientific attention not only as cell-cell communicators but also as therapeutic drugs in the regulation of tumor progression.

Here, we summarized current knowledge regarding immune cell-derived sEVs, which are secreted from several immune cells, including DCs, T cells, natural killer (NK) cells, and macrophages for cancer treatment (Fig. 1, Table 1). We also discussed some of the challenges associated with the application of immune cell-derived sEVs and future perspectives in this evolving field.

\section{CONTENTS}

\section{Dendritic cell-derived sEVs}

DCs play a key role in launching innate and adaptive immunity as antigen-presenting cells (21). Although the percentage of DCs is substantially low in the TME, it has been discovered that DCs are crucial for the start of acquired immunity in

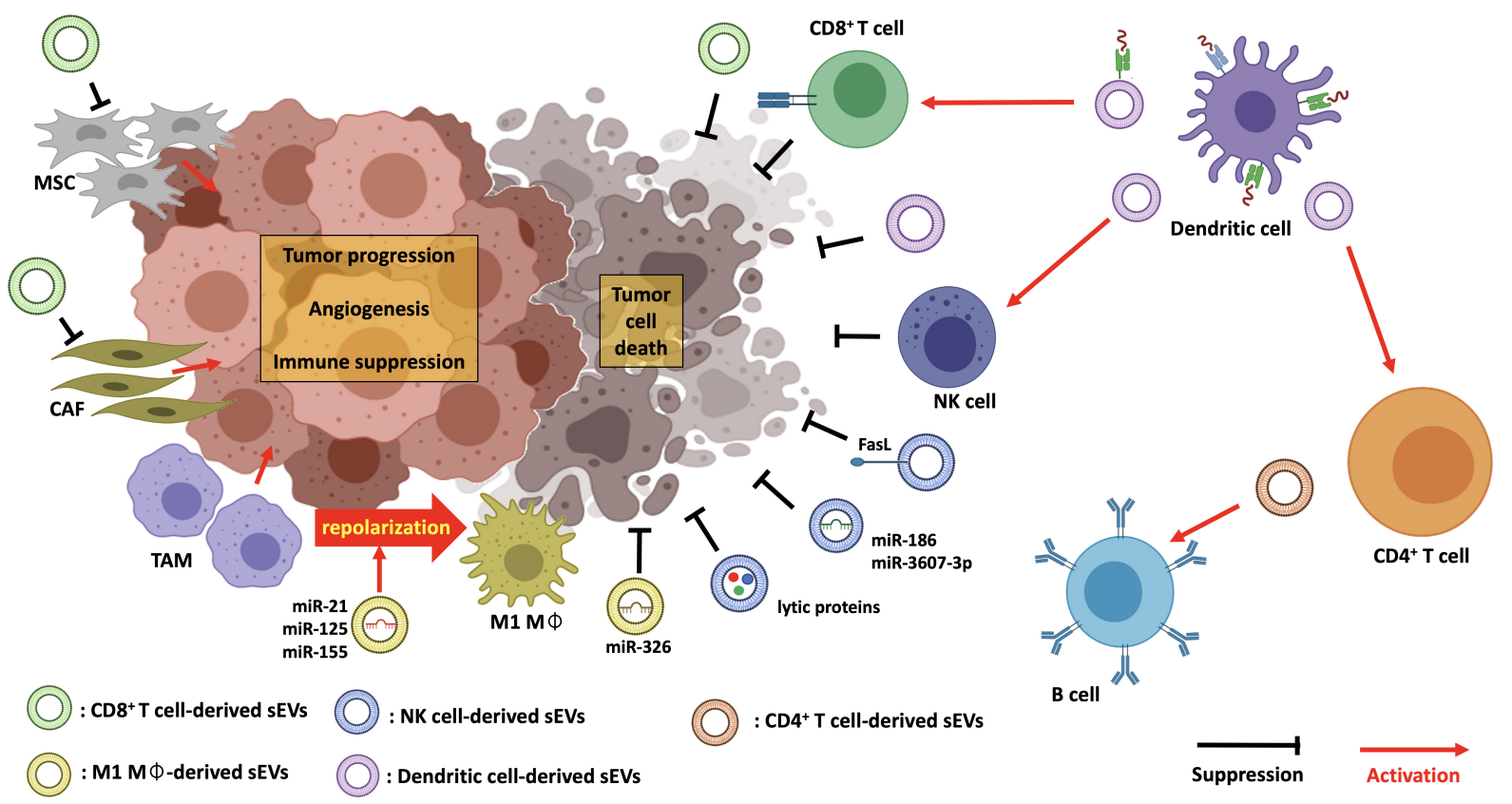

Fig. 1. Schematic diagram of immune cell-derived sEV function in tumor immune microenvironment. 


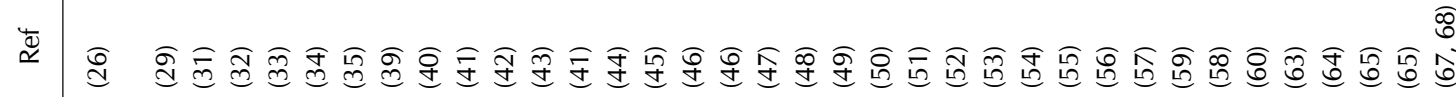
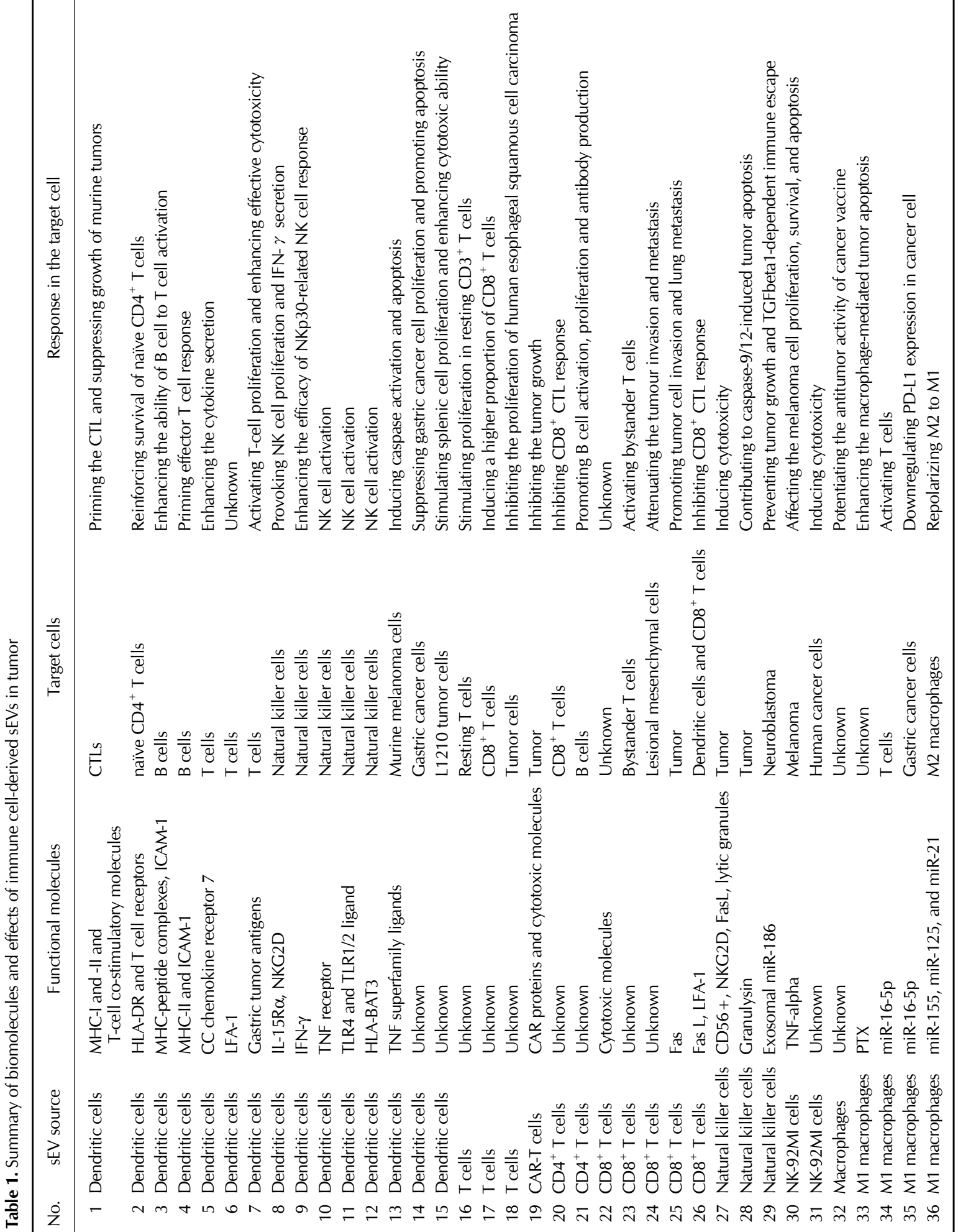
'cancer-immunity cycle', highlighting the importance of modifying DCs function to enhance cancer immunotherapy (22, 23). Traditionally, it is known that DCs uptake and process external antigens through two pathways: either displaying on the cell surface with major histocompatibility complex (MHC) molecules or completely digesting them. However, recent studies have discovered that absorbed antigen peptide presented by MHCs were also secreted with sEVs which can present antigens to other immune cells. Since the cancer vaccines are considered as cancer cell-derived proteins which can stimulate immune system, resulting in either treatment of existing cancer or prevention of cancer development $(24,25)$, it is expected that these DC-shed sEVs containing both antigen peptide and MHCs possess potential applicability as cancer vaccine. Indeed, there are several investigations revealing that $\mathrm{sEV} s$ from $D C$ present cancer antigen and exhibit high immunogenicity (26-28). Moreover, based on the fact that DC-derived SEVs are more stable and amenable to manipulate than DCs (28), many researches have been explored to utilize DC-shed sEVs for cancer treatment.

In 1998, it was first revealed that MHC and T cell co-stimulatory molecules were contained in DC-secreted sEVs and were able to activate cytotoxic T lymphocytes (CTLs), thereby suppressing murine tumor progression (26). Also, sEVs shed from monocyte-derived DCs reinforced $\mathrm{CD}^{+} \mathrm{T}$ cell survival, through interplay between $\mathrm{T}$ cell receptors and human leukocyte antigen (HLA) (29). Therefore, more detailed mechanism of interplay between DC-shed $\mathrm{sEV}$ s and immune cells contributing to reinforced immune response has been studied. To activate $T$ cells, DC-shed sEVs directly combine with $\mathrm{T}$ cells or they are indirectly transferred to APCs. Especially, B cells are largely involved in DC-secreted sEV-induced T cell activation. For the polarization of $\mathrm{T}$ cells to the Th1 type and generation of specific $T$ cell response, DC-shed sEVs depend on B cell activation (30). Furthermore, when DC-sEVs transfer both ICAM-1 and MHC-peptide complexes to B cells, the capability of B cell to activate $T$ cell is enhanced (31). Another research also proved that B cells are indispensable when CTLs are activated by DC-derived sEVs (32). These studies support the importance of $\mathrm{B}$ cell involvement in the induction of T cell via DC-shed sEVs. Other studies discovered the direct interactions between DC-shed sEVs and T cells. DC-derived sEVs were taken by T cell in the spleen by C-C chemokine receptor 7-dependant manner and enhanced the cytokine secretion (33). Also, it was revealed that LFA-1, the intermediator on $\mathrm{T}$ cell surface, mediated the recruitment of MHC-II-containing sEVs (34).

In addition to the contents of DC-shed sEVs, stimulators of DCs to secrete sEVs, like endotoxin, tumor-associated molecule, or cytokines, are important to enhance antitumor efficiency of sEVs. The lipopolysaccharide-induced mature DCs secrete sEVs with enriched MHC-II and ICAM-1. Consequently, sEVs can potently activate effector T cells in contrast to immature DCshed $\mathrm{sEV}$ s (32). Moreover, it has been revealed that only mature DCs could secrete $\mathrm{sEV}$ s which would promote the interchange of $\mathrm{MHC}$ complexes between DCs, thereby activating $\mathrm{CD}^{+} \mathrm{T}$ cells (35). Also, breast adenocarcinoma cell-treated DC-derived $\mathrm{sEV} s$ provoked more potent $\mathrm{T}$ cell response against tumor cells compared to sEVs from non-treated DCs (36). When DCs were stimulated by gastric tumor antigens, they secreted sEVs which stimulated T cell proliferation and improved cytotoxicity (37). Lastly, a recent in vivo study utilizing murine autochthonous hepatocellular carcinoma (HCC)-bearing mice model showed that treatment with $\mathrm{sEVs}$ derived from $\alpha$-fetoprotein (AFP)expressing DCs elicited tumor regression with enhanced antitumor activity and attenuated immune-suppression (38).

Similar with T cells, NK cells which recruit DC-derived sEVs exerted anti-tumor function. In melanoma cell, treatment of DC-shed sEVs to NK cell provoked proliferation and cytokine secretion via IL-15R $\alpha$ - and natural killer group 2 member D (NKG2D)-dependent manners (39). Another kind of DC-secreted $s \mathrm{EV} s$ with enriched IFN- $\gamma$ enhanced the efficacy of NK cell response against non-small cell lung cancer (40). Several other studies also proved that DC-derived $\mathrm{sEV}$ s induced NK cell activation via TNF receptor- (41), TLR4-, and TLR1/2 ligand(42), or HLA-BAT3-dependent manner (43). These findings highlight the potent abilities of DC-shed sEVs to regulate NK cells and offer novel application of DC-secreted sEVs in cancer therapy.

Besides affecting various immune cells, under some cancer conditions, it has been investigated that DC-derived sEVs could recognize and kill cancer cells. sEVs from DCs induced apoptosis in murine melanoma cell through TNF superfamily ligands present on their surface (41). Also, another study revealed that $\mathrm{sEVs}$ from hyperthermic $\mathrm{CO}_{2}$-treated DCs showed tumor regression by elevating apoptosis and inhibiting gastric cancer cell proliferation (44). Lastly, sEVs derived from DCs also exhibited cytotoxicity against L1210 tumor cells (45).

To sum up the effects of DC-derived sEVs, they participate in reinforcing immune response under various tumor conditions. DC-secreted sEVs can directly suppress tumor progression and indirectly promote immune response against tumor. Compared to DCs, DC-shed sEVs have several advantages as therapeutical agents, such as, high stability and reduced risk of in vivo replication. However, more detailed researches are needed to provide a more comprehensive understanding in terms of status of DCs and interacting molecules between cancer and DCsecreted $\mathrm{sEV}$ s for more precise application.

\section{T cell-derived sEVs}

T lymphocytes are the most abundant leukocytes and participate in various immune responses, including tumor immune response. According to their phenotype, T cells can be divided into 4 groups, namely, cytotoxic T cells, helper T cells, memory $T$ cells, and regulatory $T$ cells. Each subtype elicits specific surface markers and produces respective effector molecules. According to vigorous studies about the relationship between $\mathrm{T}$ cells and cancer cells, the role of each $\mathrm{T}$ cell and secreted molecules has been investigated as target of cancer therapies. 
Especially, the function and application of sEVs derived from $\mathrm{T}$ cells are getting attention with the expectation that $T$ cellderived sEVs would exert anti-tumor effects similar to those of their origin cells. In this review, we explained the diverse functions of sEVs from $T$ cell according to $T$ cell subtypes, based on the fact that $s E V s$ would reflect the unique function of the producer cell.

In the early stage of research on T cell-derived sEVs, the precise $T$ cell subtype that secreted $\mathrm{sEV}$ s was not characterized. It was revealed that the activated human T cells produced sEVs which could stimulate resting $\mathrm{CD}^{+}{ }^{+} \mathrm{T}$ cell proliferation (46). Along with IL-2, T cell-shed sEVs increased $\mathrm{CD}^{+}{ }^{+} \mathrm{T}$ cell population which would produce cytotoxic cytokine. Therefore, it was expected that $\mathrm{T}$ cell-derived sEVs could exhibit antitumor effect. However, it was also demonstrated that human esophageal squamous cell-infiltrating $\mathrm{T}$ cells secreted $\mathrm{sEV} s$ which promoted human esophageal squamous cell carcinoma metastasis and simultaneously inhibited the proliferation of tumor (47). Recently, chimeric antigen receptor (CAR)-containing sEVs from CAR-T cells containing high level of cytotoxic molecules inhibited tumor growth (48). These studies implicate that more detailed investigations are required to disclose the function of T cell-secreted sEVs. Classifying the T cell subtype is insufficient for unveiling the precise function of $\mathrm{T}$ cell-shed sEVs. It was revealed that $\mathrm{CD}^{+}{ }^{+} \mathrm{T}$ cell-released sEVs could restrict antitumor effect of $\mathrm{CD}^{+}{ }^{+} \mathrm{T}$ cell against melanoma cell (49). On the other hand, a recent study found that $\mathrm{CD}^{+} \mathrm{T}$ cell-released $\mathrm{sEV}$ s could promote $\mathrm{B}$ cell activation, proliferation, and antibody production, thereby enhancing antigen-specific humoral immune responses (50). Similar with the studies of $\mathrm{CD}^{+}{ }^{+}$cell-secreted sEVs, the effects of $\mathrm{CD}^{+}{ }^{+} \mathrm{T}$ cell-derived $\mathrm{sEV}$ s on tumor are not consistent. It is known that $\mathrm{CD}^{+}$ T cell-derived sEVs contain several cytotoxic molecules (51) and have the ability to either activate bystander T cells (52) or suppress lesional mesenchymal cells (53). However, other studies reported the protumor effect of $\mathrm{CD}^{+} \mathrm{T}$ cell-derived sEVs. Cai $\mathrm{Z}$ et al. showed that activated $\mathrm{CD}^{+} \mathrm{T}$ cell-derived $\mathrm{sEVs}$ promoted tumor cell invasion metastasis and exhibited little cytotoxic effect on tumor cells (54). Lastly, it was reported that $\mathrm{SEV}$ s derived from activated $\mathrm{CD}^{+} \mathrm{T}$ cells inhibited antitumor effect by decreasing the $\mathrm{MHC}$ in $\mathrm{DCs}$ and $\mathrm{CD}^{+}{ }^{+} \mathrm{T}$ cell activity in melanoma model (55).

The importance of $\mathrm{T}$ cell-secreted $\mathrm{sEV}$ s in cancer treatment has become apparent. They can function as mediator in cancerimmunity by regulating the activity of both immune and cancer cells. Furthermore, T cell-derived sEVs have strong ability to penetrate TME where T cells can hardly infiltrate. These findings indicate that not only $T$ cells but also their $\mathrm{sEV}$ s have potential to be utilized as therapeutic agents in cancer treatment. However, the duplicity of T cell-derived sEVs is a major hurdle to the precise interpretation of $\mathrm{sEV}$ function and their application in tumor treatment. Therefore, more detailed molecular mechanism of $\mathrm{T}$ cell-shed $\mathrm{sEVs}$ and engineering of $\mathrm{sEV} s$ to reinforce the natural anti-cancer function would pave the way to improve clinical efficacy.

\section{Natural killer cell-derived sEVs}

NK cells are cytotoxic innate lymphocytes mediating tumor immune surveillance and clearance. NK cells kill tumor cells by secreting cytotoxic proteins, including granzyme $\mathrm{A} / \mathrm{B}$, perforin, and granulysin. In contrast to cytotoxic adaptive lymphocytes, NK cells do not require APC-mediated antigen priming. In addition, different activating and inhibitory receptors are used in NK cells as compared to $\mathrm{CD}^{+}{ }^{+}$cells, supporting an increased interest for cancer treatment. However, solid tumors show remarkable challenges to the application of cell therapy because the solid tumor cells are difficult to infiltrate. Besides, dampening NK cell responses in TME is a hurdle. Unlike cells, nano-sized sEVs can easily diffuse and infiltrate tumors. Moreover, it has not been reported that NK cell-derived SEVs are influenced by immunosuppressive TME. Hence, NK cell-derived sEV therapy could overcome the limitations of NK cell therapy.

There is increasing evidence that NK cell-derived $s E V s$ are involved in antitumor activity. These sEVs carry bioactive molecules, such as cytotoxic proteins, cytokines, and microRNAs (miRs), which exhibit tumor-killing and immunomodulatory activities. It has been reported that sEVs derived from both resting and activated NK cells exerted comparable ex vivo cytotoxicity against hematopoietic cancer cells and solid tumor cells but not against human peripheral blood mononuclear cell (PBMC). Both sEVs expressed similar expression levels of CD56 ${ }^{+}$, NKG2D, FasL, and lytic granules (granzyme A/B and perforins). These sEVs induce tumor cell death by Fas/FasL and lytic granule-mediated apoptosis (56). sEVs isolated from ex vivoexpanded NK cells contain granulysin that contribute to caspase-9/12-induced tumor apoptosis (57). sEVs released from NK-92Ml cells expressed pro-inflammatory cytokine TNF-alpha, which affected melanoma cell proliferation, survival, and apoptosis (58). Besides proteins, miRs contribute to tumor inhibition. Exosomal miR-186 generated from ex vivo-expanded NK cells is cytotoxic to neuroblastoma. In this cancer mouse model, miR-186 prevented tumor growth and TGF beta1-dependent immune escape (59). Moreover, miR-3607-3p suppressed tumor migration and invasion by decreasing IL-26 that induced pancreatic cancer proliferation and metastasis. To improve anticancer effects, sEVs were isolated from IL-15-stimulated NK92MI. These sEVs exhibited higher cytotoxicity against human cancer cell lines without affecting normal human cells (60).

Taken together, it is obvious that NK cell-derived sEVs exert cancer-specific cytotoxicity. Given that sEVs are able to cross the blood-brain barrier (BBB), NK cell-derived sEVs could be utilized in glioblastoma treatment. Moreover, sEVs overcome the limitations of NK cell therapy. Therefore, NK cell-derived sEV-based therapy could be regarded as a major cancer therapy in the future.

\section{Macrophage-derived sEVs}

Macrophages are mainly classified into two different polariza- 
tion states, namely, pro-inflammatory M1 type and anti-inflammatory M2 type macrophages. While M2 type tumor-associated macrophages (TAMs) promote angiogenesis, tumor growth, invasion, metastasis, and drug resistance (61), M1 macrophages kill tumor cells by releasing tumor killing molecules, including, reactive oxygen species (ROS), nitric oxide synthase (iNOS), and pro-inflammatory cytokines (62). Thus, M1 macrophagederived sEVs (M1-sEVs) could be promising sEVs for anti-cancer therapy.

Like M1 macrophages, M1-sEVs enhance anti-tumor immunity by generating pro-inflammatory environment. After exposure of macrophages, $\mathrm{T}$ cells, and DCs in M1-sEVs, these cells increase expression levels of pro-inflammatory cytokines. sEVs isolated from INF-gamma-stimulated macrophages can potentiate the antitumor activity of cancer vaccine by providing a pro-inflammatory microenvironment in the lymph node (63). M1-sEV-treated macrophages also enhance caspase-3-mediated tumor apoptosis. The anti-tumor effects of paclitaxel (PTX) were substantially improved when PTX was loaded into M1-sEVs (64). M1-sEVs also suppress gastric cancer (GC) by activating T cells and downregulating PD-L1 expression in cancer cells. miR-16-5p expressed in M1-sEVs mainly contributes to decrease the level of PD-L1 in GC, and increases $\mathrm{CD}^{+}{ }^{+}$and INF-gamma ${ }^{+} \mathrm{T}$ cells (65). Moreover, M1-sEVs suppress hepatocellular carcinoma cell (HCC) progression by decreasing NFkappaB expression in HCC cells.

In TME, M2 tumor-associated macrophages (TAMs) promote tumor growth and suppress antitumor immune responses by releasing angiogenic factors and anti-inflammatory cytokines (66). Thus, repolarization of M2 TAMs to M1 macrophages in TME is critical for cancer therapies. Interestingly, M1-sEVs induce the differentiation of native monocytes or M2 macrophages into activated macrophages (M1). M1-sEV treatment successfully reverts $M 2$ to $M 1$ macrophages in tumor tissues. Intravenous injection of M1-sEVs into tumor-bearing mice suppressed tumor growth. Enrichment of M1 polarization-inducing miRs including miR-155, miR-125, and miR-21 in M1-sEVs addresses the capability of repolarization of M2 to M1 macrophages $(67,68)$.

Taken together, M1-sEVs are attractive anti-cancer therapeutics, providing pro inflammatory environment. Moreover, miRs derived from $\mathrm{M} 1$-sEVs play key roles in $\mathrm{M} 2$ to $\mathrm{M} 1$ repolarization and downregulation of PD-L1 in GC cells. It is expected that a variety of miRs derived from M1-sEVs would be identified as anti-cancer active molecules.

\section{DISCUSSION}

Recently, immunotherapy was emerged as an innovative cancer treatment to strengthen our immune system. Immunotherapy is classified into cellular immunotherapy, immune checkpoint inhibitors, cytokine therapy, and sEV-based immunotherapy. sEV immunotherapy has various advantages including biocompatibility, ideal bio-active molecules' carrier, BBB penetration, and their small size suitable for infiltration into solid tumors. In particular, sEV therapy could overcome the huddles of cell therapy. For example, immune cells are suppressed in TME and cannot easily infiltrate tumor tissues. On the other hand, sEVs can easily infiltrate tumor tissues without being affected by the TME. Moreover, combinational therapy of sEVs with other immunotherapies or chemotherapies potentiate efficacy. Hence, sEV-based immunotherapy is a very promising therapy.

In this review, we focused on immune cell-derived sEVs for immune cancer therapy. In contrast to cancer cell-derived sEVs, immune cell-derived sEVs exhibited anti-cancer effects. Dendritic cell-derived sEVs were developed as cancer vaccines. CAR-T cell-derived sEVs and NK cell-derived sEVs exerted cytotoxicity against cancer cells. CD8 ${ }^{+} \mathrm{T}$ cell-derived sEVs inhibited tumor-associated cells including CAF and MCS. M1-sEVs provided proinflammatory environment along with $M 2$ to $M 1$ repolarization. However, some immune cell-derived sEVs were not yet studied for application as sEV-based immunotherapy. For instance, reports of $\mathrm{sEV}$ s derived from $\mathrm{B}$ cells and mast cells are scarce. In case of mast cell-derived sEVs, they are uncertain for anti-cancer therapeutics because mast cells are double-edged sword in cancer therapy. While some components of mast cells induce angiogenesis and cancer shaping, their proinflammatory cytokines suppressed tumor progression (69). Therefore, further research is required on SEVs secreted from B cells, neutrophils, and mast cells to broaden sEV-based therapy.

In spites of many advantages of immune cell-derived sEVs for anti-cancer therapy, off-target effects raise concerns on clinical application. Firstly, excessive amounts of cytotoxic internal contents of sEVs lead to off-target toxicity. Although it

Table 2. Clinical trials of immune cell-derived sEVs for cancer treatment

\begin{tabular}{|c|c|c|c|c|}
\hline sEV source & Study title & $\begin{array}{l}\text { Treated cancer } \\
\text { type }\end{array}$ & Status & Ref \\
\hline $\begin{array}{l}1 \text { Dendritic cells } \\
\text { (autologous PBMCs) }\end{array}$ & $\begin{array}{l}\text { Vaccination of metastatic melanoma patients with autologous dendritic cell (DC) } \\
\text { derived-exosomes: results of the first phase I clinical trial }\end{array}$ & Melanoma & $\begin{array}{l}\text { Phase } 1 \\
\text { (terminated) }\end{array}$ & 72 \\
\hline 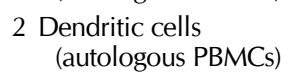 & $\begin{array}{l}\text { A phase I study of dexosome immunotherapy in patients with } \\
\text { advanced non-small cell lung cancer }\end{array}$ & $\begin{array}{l}\text { Non-small cell } \\
\text { lung carcinoma }\end{array}$ & $\begin{array}{l}\text { Phase } 1 \\
\text { (terminated) }\end{array}$ & 40 \\
\hline $\begin{array}{l}3 \text { Dendritic cells } \\
\text { (autologous PBMCs) }\end{array}$ & $\begin{array}{l}\text { Dendritic cell-derived exosomes as maintenance immunotherapy after } \\
\text { first line chemotherapy in NSCLC }\end{array}$ & $\begin{array}{l}\text { Non-small cell } \\
\text { lung carcinoma }\end{array}$ & $\begin{array}{l}\text { Phase } 2 \\
\text { (terminated) }\end{array}$ & 73 \\
\hline
\end{tabular}


has been no report that intrinsic sEVs derived from NK cells, DC cells, $T$ cells and macrophages induce serious off-target toxicity, off-target effect could be observed in high does SEV treatment. Secondly, immune cell-derived sEVs could induce cytokine release syndrome (CRS) because they provide proinflammatory environment. Until now, there is no document reporting that intrinsic immune cell-derived sEVs cause CRS. However, high does may induce CRS. Thirdly, sEV contents and their effects on tissues and immune system were partially understood. In-depth studies are required such as proteomic and miR profiling along with their functions on various tissues and immune system. Collectively, we should carefully check the sEV does to avoid off-target effects for clinical application.

In the pharmaceutical industry, large-scale production and quality control are very important. For industrial application of $\mathrm{sEVs}$, large scale production of immune cell-derived $\mathrm{sEV} s$ is required. Some cytokines and feeder cells are helpful to increase the yield of immune-derived sEVs. In case of NK cell-derived sEVs, K562 cells expressing membrane-bound IL-21 dramatically increased the yield of NK-derived sEVs by improving NK cell proliferation (70). Regarding the increase in the yield of $\mathrm{CD}^{+}{ }^{+} \mathrm{T}$ cell-derived sEVs, IL-12 could be utilized for cell culture (52). sEV yield could also be improved by sEV purification method. Tangential flow filtration (TFF) is an emerging technique to concentrate $s E V s$ with permeable membrane filtration and 'tangential flow'. This method is more time-efficient and the yield of sEVs could be scaled up with less aggregates as compared to ultracentrifugation (71). Next, quality control of $\mathrm{sEVs}$ is required. In other words, the level of anti-cancer activity should be maintained during sEV production. Cell culture conditions should be accurately controlled to get the same quality of $\mathrm{sEV}$ s.

To increase the efficacy of immune-derived sEVs, sEV engineering or generation of $\mathrm{sEV}$ s from cytokine-stimulated cells could be considered. Firstly, CAR-T cell-derived sEVs expressed chimeric antigen receptors on their surface. These receptors enabled $\mathrm{sEV}$ s to specifically recognize cancer cells. In addition, co-stimulating domains were harbored in these receptors to evaluate anti-cancer activity (48). Therefore, antibody expressed on sEV surface could be utilized for cancer targeting. Moreover, expression of proper co-stimulators could augment their anticancer activity. Secondly, immune-derived sEVs could improve their anti-cancer activity by cytokine stimulation. For example, sEVs derived from IL-15-stimulated NK cells enhanced antitumor potency (60). In addition to NK-sEVs, other immune cell-derived sEVs could enhance anti-cancer effect by generating sEVs derived from appropriate cytokine-treated immune cells.

Recently, there were clinical trials of immune cell-derived sEVs for cancer treatment (Table 2). DC-derived sEVs with tumor antigen were treated as cancer vaccines. Phase I and II clinical trials were launched for melanoma and non-small cell lung cancer, respectively. But these clinical experiences showed limited efficacy, despite of the outstanding results in preclinical studies $(40,72,73)$. sEVs derived from other immune cells including T cells, NK cells, and macrophages should be tested for clinical use of cancer immunotherapy.

Taken together, the advantages mentioned above support $\mathrm{sEV}$-based therapy as a promising immunotherapy. First of all, immune cell-derived sEVs contain natural anti-cancer contents. In the future, $\mathrm{sEV}$ engineering or producing $\mathrm{SEV}$ s secreted from cytokine-stimulated immune cells could enhance antitumor potency. These sEVs could be utilized against various types of tumors especially solid and brain tumors.

\section{ACKNOWLEDGEMENTS}

This research was supported by the Bio \& Medical Technology Development Program of the National Research Foundation (NRF) of Korea funded by the Ministry of Science \& ICT (2017 M3A9G8083382 and 2020M3A9I4039539), the National Research Foundation of Korea (NRF) grant funded by the Korea government (MSIT) (No.2021R1A5A2021614), and the DGIST Start-up Fund Program of the Ministry of Science and ICT (2018010109).

\section{CONFLICTS OF INTEREST}

The authors have no conflicting interests.

\section{REFERENCES}

1. Thery C, Witwer KW, Aikawa E et al (2018) Minimal information for studies of extracellular vesicles 2018 (MISEV 2018): a position statement of the International Society for Extracellular Vesicles and update of the MISEV2014 guidelines. J Extracell Vesicles 7, 1535750

2. Kalluri R and LeBleu VS (2020) The biology, function, and biomedical applications of exosomes. Science 367, eaau6977

3. Thery C, Zitvogel L and Amigorena S (2002) Exosomes: composition, biogenesis and function. Nat Rev Immunol 2, 569-579

4. Kahlert C and Kalluri R (2013) Exosomes in tumor microenvironment influence cancer progression and metastasis. J Mol Med (Berl) 91, 431-437

5. Mathieu M, Martin-Jaular L, Lavieu G and Thery C (2019) Specificities of secretion and uptake of exosomes and other extracellular vesicles for cell-to-cell communication. Nat Cell Biol 21, 9-17

6. Bebelman MP, Smit MJ, Pegtel DM and Baglio SR (2018) Biogenesis and function of extracellular vesicles in cancer. Pharmacol Ther 188, 1-11

7. Ciardiello C, Cavallini L, Spinelli C et al (2016) Focus on extracellular vesicles: new frontiers of cell-to-cell communication in cancer. Int J Mol Sci 17, 175

8. van Niel G, D'Angelo G and Raposo G (2018) Shedding light on the cell biology of extracellular vesicles. Nat Rev Mol Cell Biol 19, 213-228

9. Urbanelli L, Magini A, Buratta S et al (2013) Signaling pathways in exosomes biogenesis, secretion and fate. Genes (Basel) 4, 152-170

10. McLellan AD (2009) Exosome release by primary B cells. 
Crit Rev Immunol 29, 203-217

11. Segura E, Amigorena S and Thery C (2005) Mature dendritic cells secrete exosomes with strong ability to induce antigen-specific effector immune responses. Blood Cells Mol Dis 35, 89-93

12. Paolillo M and Schinelli S (2017) Integrins and exosomes, a dangerous liaison in cancer progression. Cancers (Basel) 9

13. Segura E, Guerin C, Hogg N, Amigorena S and Thery C (2007) CD8 + dendritic cells use LFA-1 to capture MHCpeptide complexes from exosomes in vivo. J Immunol 179, 1489-1496

14. Kalluri R (2016) The biology and function of exosomes in cancer. J Clin Invest 126, 1208-1215

15. Gao D and Jiang L (2018) Exosomes in cancer therapy: a novel experimental strategy. Am J Cancer Res 8, 2165-2175

16. Batrakova EV and Kim MS (2015) Using exosomes, naturally-equipped nanocarriers, for drug delivery. J Control Release 219, 396-405

17. Kibria G, Ramos EK, Wan Y, Gius DR and Liu H (2018) Exosomes as a drug delivery system in cancer therapy: potential and challenges. Mol Pharm 15, 3625-3633

18. Yong T, Zhang X, Bie N et al (2019) Tumor exosomebased nanoparticles are efficient drug carriers for chemotherapy. Nat Commun 10, 3838

19. Li Y, Wan YY and Zhu B (2017) Immune cell metabolism in tumor microenvironment. Adv Exp Med Biol 1011, 163196

20. Guo S and Deng CX (2018) Effect of stromal cells in tumor microenvironment on metastasis initiation. Int J Biol Sci 14, 2083-2093

21. Steinman RM (2012) Decisions about dendritic cells: past, present, and future. Annu Rev Immunol 30, 1-22

22. Pitt JM, Charrier M, Viaud S et al (2014) Dendritic cellderived exosomes as immunotherapies in the fight against cancer. J Immunol 193, 1006-1011

23. Chen DS and Mellman I (2013) Oncology meets immunology: the cancer-immunity cycle. Immunity 39, 1-10

24. Saxena M, van der Burg SH, Melief CJM and Bhardwaj N (2021) Therapeutic cancer vaccines. Nat Rev Cancer 21, 360-378

25. Santos P and Almeida F (2021) Exosome-based vaccines: history, current state, and clinical trials. Front Immunol 12,711565

26. Zitvogel L, Regnault A, Lozier A et al (1998) Eradication of established murine tumors using a novel cell-free vaccine: dendritic cell-derived exosomes. Nat Med 4, 594-600

27. Andre F, Chaput N, Schartz NE et al (2004) Exosomes as potent cell-free peptide-based vaccine. I. Dendritic cellderived exosomes transfer functional MHC class I/peptide complexes to dendritic cells. J Immunol 172, 2126-2136

28. Pitt JM, Andre F, Amigorena S et al (2016) Dendritic cell-derived exosomes for cancer therapy. J Clin Invest $126,1224-1232$

29. Matsumoto K, Morisaki T, Kuroki H et al (2004) Exosomes secreted from monocyte-derived dendritic cells support in vitro naive $\mathrm{CD} 4+\mathrm{T}$ cell survival through NF-(kappa)B activation. Cell Immunol 231, 20-29

30. Qazi KR, Gehrmann U, Domange Jordo E, Karlsson MC and Gabrielsson S (2009) Antigen-loaded exosomes alone induce Th1-type memory through a B-cell-dependent mecha- nism. Blood 113, 2673-2683

31. Segura E, Nicco C, Lombard B et al (2005) ICAM-1 on exosomes from mature dendritic cells is critical for efficient naive T-cell priming. Blood 106, 216-223

32. Naslund TI, Gehrmann U, Qazi KR, Karlsson MC and Gabrielsson S (2013) Dendritic cell-derived exosomes need to activate both $\mathrm{T}$ and $\mathrm{B}$ cells to induce antitumor immunity. J Immunol 190, 2712-2719

33. Wei G, Jie Y, Haibo L et al (2017) Dendritic cells derived exosomes migration to spleen and induction of inflammation are regulated by CCR7. Sci Rep 7, 42996

34. Nolte-'t Hoen EN, Buschow SI, Anderton SM, Stoorvogel $\mathrm{W}$ and Wauben $\mathrm{MH}$ (2009) Activated T cells recruit exosomes secreted by dendritic cells via LFA-1. Blood $113,1977-1981$

35. Thery C, Duban L, Segura E, Veron P, Lantz O and Amigorena S (2002) Indirect activation of naive CD4 + T cells by dendritic cell-derived exosomes. Nat Immunol 3, 1156-1162

36. Romagnoli GG, Zelante BB, Toniolo PA, Migliori IK and Barbuto JA (2014) Dendritic cell-derived exosomes may be a tool for cancer immunotherapy by converting tumor cells into immunogenic targets. Front Immunol 5, 692

37. Guan S, Li Q, Liu P, Xuan X and Du Y (2014) Umbilical cord blood-derived dendritic cells loaded with BGC823 tumor antigens and DC-derived exosomes stimulate efficient cytotoxic T-lymphocyte responses and antitumor immunity in vitro and in vivo. Cent Eur J Immunol 39, $142-151$

38. Lu Z, Zuo B, Jing R et al (2017) Dendritic cell-derived exosomes elicit tumor regression in autochthonous hepatocellular carcinoma mouse models. J Hepatol 67, 739-748

39. Viaud S, Terme M, Flament C et al (2009) Dendritic cellderived exosomes promote natural killer cell activation and proliferation: a role for NKG2D ligands and IL-15Ralpha. PLoS One 4, e4942

40. Besse B, Charrier M, Lapierre V et al (2016) Dendritic cell-derived exosomes as maintenance immunotherapy after first line chemotherapy in NSCLC. Oncoimmunology 5, e1071008

41. Munich S, Sobo-Vujanovic A, Buchser WJ, Beer-Stolz D and Vujanovic NL (2012) Dendritic cell exosomes directly kill tumor cells and activate natural killer cells via TNF superfamily ligands. Oncoimmunology 1, 1074-1083

42. Sobo-Vujanovic A, Munich S and Vujanovic NL (2014) Dendritic-cell exosomes cross-present Toll-like receptor-ligands and activate bystander dendritic cells. Cell Immunol 289, 119-127

43. Simhadri VR, Reiners KS, Hansen HP et al (2008) Dendritic cells release HLA-B-associated transcript-3 positive exosomes to regulate natural killer function. PLoS One 3, e3377

44. Wang J, Wang Z, Mo Y, Zeng Z, Wei P and Li T (2015) Effect of hyperthermic CO2-treated dendritic cell-derived exosomes on the human gastric cancer AGS cell line. Oncol Lett 10, 71-76

45. Guo F, Chang CK, Fan $\mathrm{HH}$ et al (2008) Anti-tumour effects of exosomes in combination with cyclophosphamide and polyinosinic-polycytidylic acid. J Int Med Res 36, 13421353

46. Wahlgren J, Karlson Tde L, Glader P, Telemo E and Valadi 
H (2012) Activated human T cells secrete exosomes that participate in IL-2 mediated immune response signaling. PLoS One 7, e49723

47. Min H, Sun X, Yang X et al (2018) Exosomes derived from irradiated esophageal carcinoma-infiltrating $T$ cells promote metastasis by inducing the epithelial-mesenchymal transition in esophageal cancer cells. Pathol Oncol Res 24, 11-18

48. Fu W, Lei C, Liu S et al (2019) CAR exosomes derived from effector CAR-T cells have potent antitumour effects and low toxicity. Nat Commun 10, 4355

49. Zhang $\mathrm{H}$, Xie $\mathrm{Y}$, Li W, Chibbar R, Xiong $\mathrm{S}$ and Xiang J (2011) CD4(+) T cell-released exosomes inhibit CD8(+) cytotoxic T-lymphocyte responses and antitumor immunity. Cell Mol Immunol 8, 23-30

50. Lu J, Wu J, Xie F et al (2019) CD4(+) T cell-released extracellular vesicles potentiate the efficacy of the $\mathrm{HBsAg}$ vaccine by enhancing B cell responses. Adv Sci (Weinh) 6, 1802219

51. Peters PJ, Borst J, Oorschot $V$ et al (1991) Cytotoxic $T$ lymphocyte granules are secretory lysosomes, containing both perforin and granzymes. J Exp Med 173, 1099-1109

52. Li L, Jay SM, Wang Y, Wu SW and Xiao Z (2017) IL-12 stimulates CTLs to secrete exosomes capable of activating bystander CD8(+) T cells. Sci Rep 7, 13365

53. Seo N, Shirakura $Y$, Tahara $Y$ et al (2018) Activated CD8(+) T cell extracellular vesicles prevent tumour progression by targeting of lesional mesenchymal cells. Nat Commun 9, 435

54. Cai Z, Yang F, Yu L et al (2012) Activated T cell exosomes promote tumor invasion via Fas signaling pathway. J Immunol 188, 5954-5961

55. Xie $Y$, Zhang H, Li W et al (2010) Dendritic cells recruit $T$ cell exosomes via exosomal LFA-1 leading to inhibition of $\mathrm{CD} 8+\mathrm{CTL}$ responses through downregulation of peptide/MHC class I and Fas ligand-mediated cytotoxicity. J Immunol 185, 5268-5278

56. Lugini L, Cecchetti S, Huber V et al (2012) Immune surveillance properties of human NK cell-derived exosomes. J Immunol 189, 2833-2842

57. Jong $\mathrm{AY}, \mathrm{Wu} \mathrm{CH}$, Li J et al (2017) Large-scale isolation and cytotoxicity of extracellular vesicles derived from activated human natural killer cells. J Extracell Vesicles 6, 1294368

58. Zhu L, Kalimuthu S, Gangadaran P et al (2017) Exosomes derived from natural killer cells exert therapeutic effect in melanoma. Theranostics 7, 2732-2745

59. Neviani P, Wise PM, Murtadha M et al (2019) Natural killer-derived exosomal miR-186 inhibits neuroblastoma growth and immune escape mechanisms. Cancer Res 79,
1151-1164

60. Zhu L, Kalimuthu S, Oh JM et al (2019) Enhancement of antitumor potency of extracellular vesicles derived from natural killer cells by IL-15 priming. Biomaterials 190-191, 38-50

61. Ngambenjawong C, Gustafson HH and Pun SH (2017) Progress in tumor-associated macrophage (TAM)-targeted therapeutics. Adv Drug Deliv Rev 114, 206-221

62. Bernsmeier C, van der Merwe S and Perianin A (2020) Innate immune cells in cirrhosis. J Hepatol 73, 186-201

63. Cheng L, Wang $Y$ and Huang $L$ (2017) Exosomes from M1-polarized macrophages potentiate the cancer vaccine by creating a pro-inflammatory microenvironment in the lymph node. Mol Ther 25, 1665-1675

64. Wang P, Wang H, Huang Q et al (2019) Exosomes from M1-polarized macrophages enhance paclitaxel antitumor activity by activating macrophages-mediated inflammation. Theranostics 9, 1714-1727

65. Li Z, Suo B, Long G et al (2020) Exosomal miRNA-16-5p derived from M1 macrophages enhances $T$ cell-dependent immune response by regulating PD-L1 in gastric cancer. Front Cell Dev Biol 8, 572689

66. Lin Y, Xu J and Lan H (2019) Tumor-associated macrophages in tumor metastasis: biological roles and clinical therapeutic applications. J Hematol Oncol 12, 76

67. Wu XQ, Dai $Y$, Yang $Y$ et al (2016) Emerging role of microRNAs in regulating macrophage activation and polarization in immune response and inflammation. Immunology 148, 237-248

68. Choo YW, Kang M, Kim HY et al (2018) M1 macrophagederived nanovesicles potentiate the anticancer efficacy of immune checkpoint inhibitors. ACS Nano 12, 8977-8993

69. Derakhshani A, Vahidian F, Alihasanzadeh M, Mokhtarzadeh A, Lotfi Nezhad P and Baradaran B (2019) Mast cells: a double-edged sword in cancer. Immunol Lett 209, 28-35

70. Farcas M and Inngjerdingen M (2020) Natural killer cellderived extracellular vesicles in cancer therapy. Scand J Immunol 92, e12938

71. Busatto S, Vilanilam G, Ticer T et al (2018) Tangential flow filtration for highly efficient concentration of extracellular vesicles from large volumes of fluid. Cells 7,273

72. Escudier B, Dorval T, Chaput N et al (2005) Vaccination of metastatic melanoma patients with autologous dendritic cell (DC) derived-exosomes: results of thefirst phase I clinical trial. J Transl Med 3, 10

73. Morse MA, Garst J, Osada T et al (2005) A phase I study of dexosome immunotherapy in patients with advanced non-small cell lung cancer. J Transl Med 3, 9 